\title{
W-band Extended Interaction Oscillations using Post-accelerated Pseudospark-sourced Electron Beams
}

\author{
A.W. Cross, H. Yin, W. He, L. Zhang, \\ K. Ronald and A.D.R. Phelps \\ Department of Physics \\ SUPA, University of Strathclyde \\ Glasgow G4 0NG, UK \\ a.w.cross@strath.ac.uk \\ G. Shu, Y. Yin \\ School of Physical Electronics \\ UESTC, Chengdu, China
}

\author{
X. Chen \\ School of Electronic Engineering \\ \& Computer Science \\ Queen Mary, \\ University of London, London \\ J. Zhao \\ School of Electrical Engineering \\ Xi'an Jiaotong University \\ Xi'an, Shaanxi Province, China
}

\begin{abstract}
This article presents the investigation of a millimetre-wave extended interaction oscillation using both pencil and sheet-shaped pseudospark (PS)-sourced electron beams. Two W-band (75-110 GHz) pencil and sheet beam extended interaction oscillators (EIO) were designed and constructed respectively. The PS-sourced pencil beam EIO structure was firstly driven by a four-gap PS discharge operating at $30.5 \mathrm{kV}$ and the output power of $38 \mathrm{~W}$ was achieved. The same EIO structure was then driven by an improved PS-sourced beam produced by combining a single-gap PS structure with an integrated post-acceleration section which generated an output power of $200 \mathrm{~W}$. The preliminary PS-sourced sheet beam EIO structure will also be presented.
\end{abstract}

Keywords-millimetre-wave; EIO; pseudospark discharge; electron beam; post-acceleration

\section{INTRODUCTION}

Millimetre-wave sources in the frequency range from 0.1 to $1 \mathrm{THz}$ have received increasing interest in recent years because of their many exciting applications such as in next $5 \mathrm{G}$ beyond high speed mobile wireless communications, new generation of electron spin resonance spectroscopy, remote imaging, radar and applications to the internet of things. To date, vacuum electronic technology still remains the main method to achieve kilowatt level high power broadband millimetre-wave radiation. Nevertheless in vacuum electronic devices high quality intense electron beams have been crucial as the frequency increases, thus the PS discharge has attracted a lot of attention recently as a promising source of high quality high intensity electron beam pulses with the beam current density up to $10^{6} \mathrm{Am}^{-2}$ and brightness up to $10^{12} \mathrm{Am}^{-2} \mathrm{rad}^{-2}$ [19]. This is because a PS-sourced electron beam has the ability to self-focus due to the unique discharge structure and the formation of an ion channel generated by the beam front. This makes it an excellent electron beam source for desirable compact millimetre-wave devices. Among various vacuum electronic devices, the EIO as a linear beam vacuum device has gained considerable attention as a promising millimetrewave oscillation source due to its high gain per unit length and compact configuration [10-12].

In this paper, we use a PS-sourced electron beam instead of the conventional electron beam produced by a thermionic cathode to drive the EIO to achieve more compact structures. To achieve better beam quality, the PS-sourced electron beam is also investigated with post-acceleration to reduce its beam energy spread $[13,14]$. To achieve higher output power, a planar EIO structure is further studied and powered by a PSsourced sheet beam [15].

\section{PS E-BEAMS \& EIO EXPERIMENTS}

Fig.1 shows the schematic PS beam extraction and EIO experimental setups of both a four-gap PS discharge and a one-gap PS discharge combining with an integrated postacceleration section to drive both the pencil beam EIO structure and the sheet beam EIO structure, where the micro pencil beam and sheet beam are obtained by applying appropriate collimators attached to the anode. Fig. 2 shows a photograph of the W-band oscillator based on a PS-sourced electron beam and the EIO circuit. It does not need an external focusing magnetic field and is capable of operating at high repetition rate (up to a few $\mathrm{kHz}$ ). Fig.3a shows a typical pencil beam current recording from the four-gap PS discharge configuration, in which it can been seen that the electron beam has bigger energy spread due to its corresponding to the 
sweeping voltage. Fig. $3 \mathrm{~b}$ presents a typical electron beam current recording versus a flatter pulsed voltage from the single-gap PS discharge with post acceleration unit, resulting in smaller beam energy spread. Fig. 4 shows a beam image captured after post acceleration.
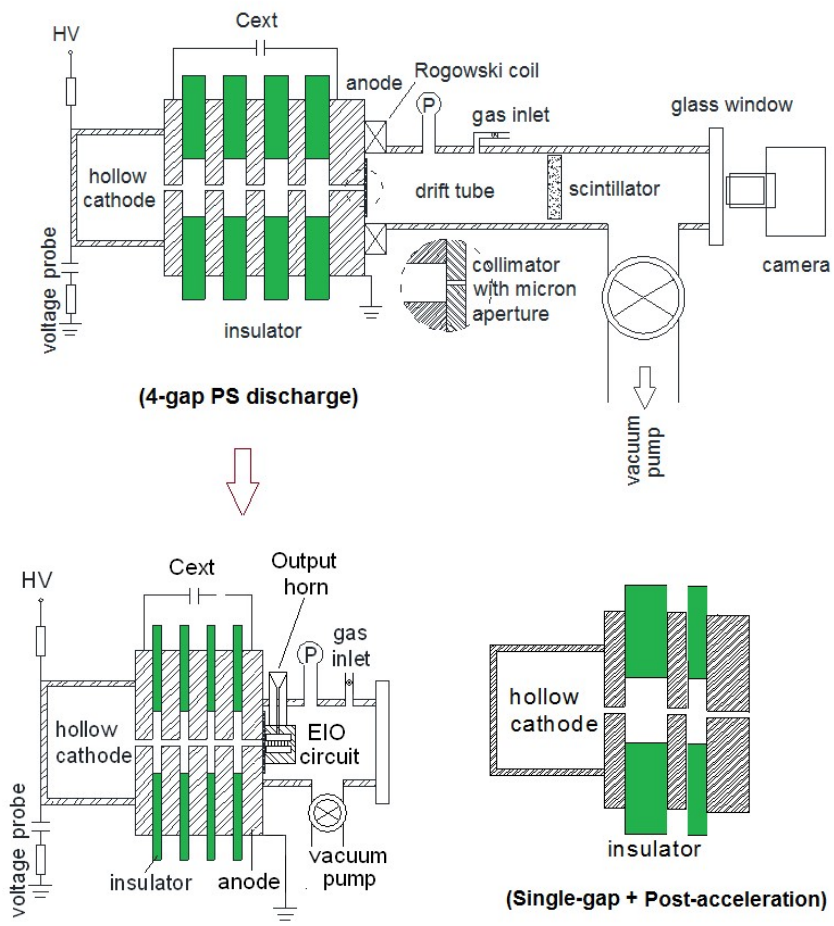

(Single-gap + Post-acceleration)

Fig.1 Schematic PS beam extraction and EIO experimental setups of both a four-gap PS discharge and a one-gap PS discharge combining with an integrated post-acceleration section

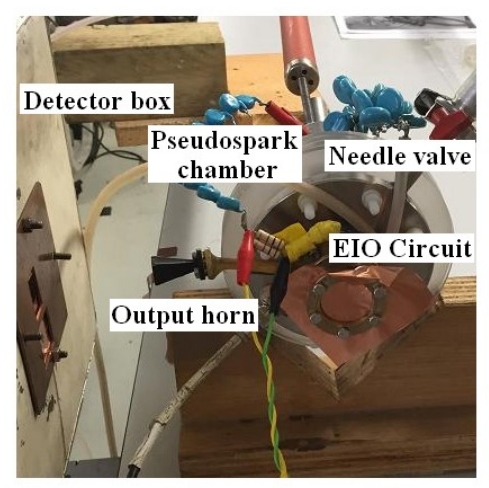

Fig.2 A photo of the EIO experimental setup

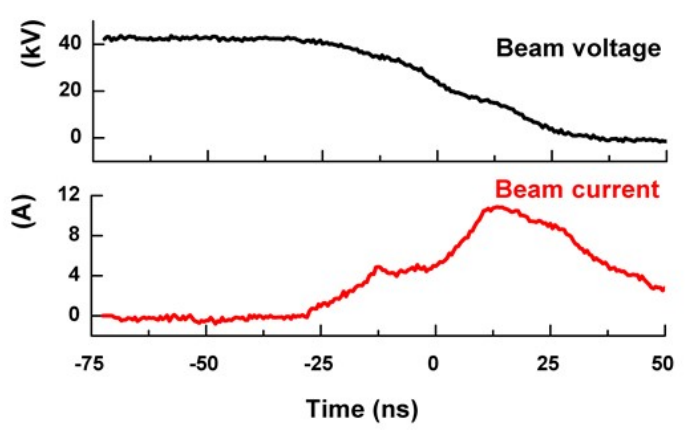

(a)



(b)

Fig.3 (a) Typical pencil beam current recordings from the four-gap PS discharge configuration, and (b) single-gap PS discharge with postacceleration section.

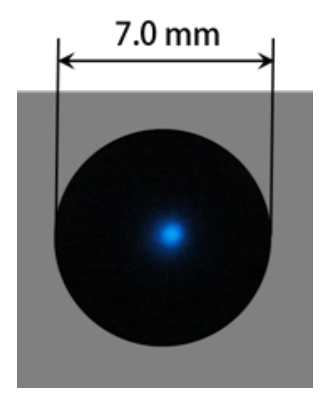

Fig. 4 PS-beam image captured after post-acceleration

A W-band $(75 \mathrm{GHz}-110 \mathrm{GHz}$ ) EIO circuit operated in the $2 \pi$ mode was selected for the first experiment because of its shorter interaction length, high interaction impedance and high gain per unit length, as compared with a backward wave oscillator. The beam voltage was designed to be centered at $30.5 \mathrm{kV}$, which is suitable for a four-gap PS discharge. This newly developed device combines the merit of a short interaction circuit in the EIO and the high current density property of the PS-sourced electron beam to generate W-band coherent radiation. Fig. 5 shows the layout, structure and dispersion of the EIO circuit, from which the EIO device and operating frequency can be seen. 


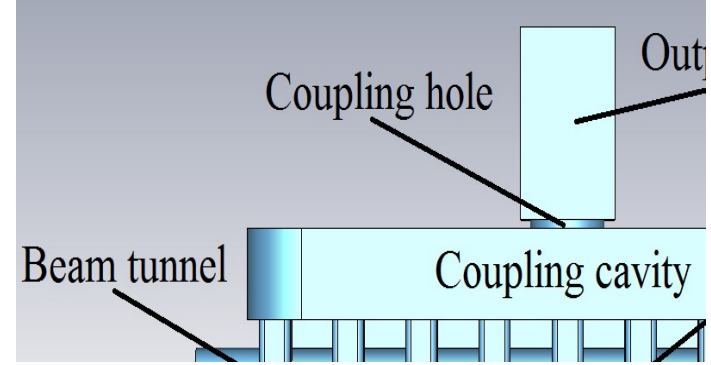

(a)

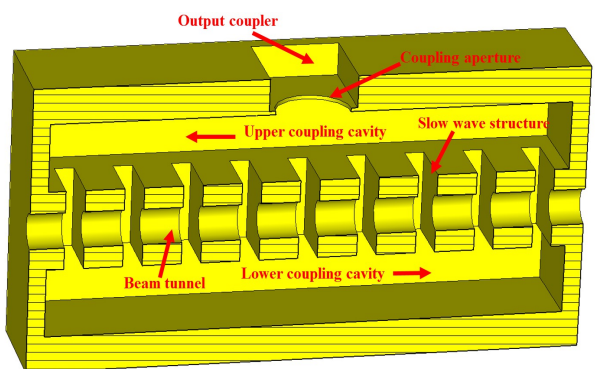

(b)

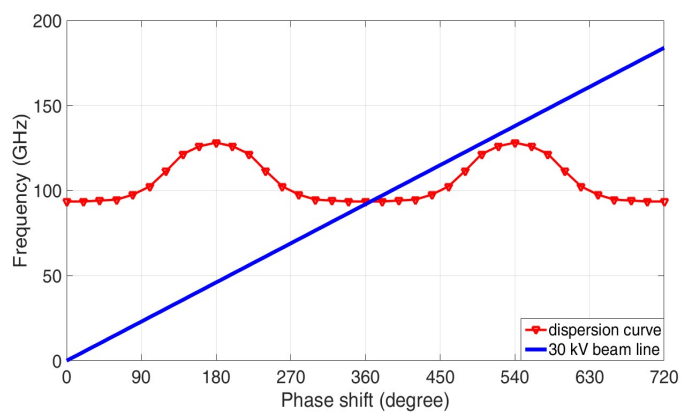

(c)

Fig. 5 (a) The layout, (b) structure and (c) dispersion of the EIO

Fig. 6 shows a photograph of the built EIO. Experimental results from the four-gap PS discharge configuration show that with a $35 \mathrm{kV}$ discharge voltage, the EIO successfully produced $\mathrm{W}$-band radiation pulses as in Fig. 7a with $38 \mathrm{~W}$ peak power and $20 \mathrm{~ns}$ duration, which agrees well with the 3D Particle-in-Cell (PIC) simulations using MAGIC. The output power was measured using the general antenna theorem with the total power from a launching antenna, calculated by integrating its radiated power density over space.



Fig.6 Photograph of the built EIO



(a)



(b)

Fig.7 (a) W-band pencil beam EIO output recordings from the four-gap PS beam, (b) and single-gap post-acceleraated PS beam.

Experiments with the same W-band pencil beam EIO structure, produced an output power of up to $200 \mathrm{~W}$ when the post-accelerated configuration in Fig. $7 \mathrm{~b}$ was used as compared to $38 \mathrm{~W}$ generated from the 4-gap PS discharge configuration shown in Fig. 7a [11]. As for the sheet beam EIO structure, simulations predict a much higher output power can be achieved. Further studies of a planar EIO with a PS sheet electron beam will be conducted using this improved configuration and a higher frequency $200 \mathrm{GHz}$ EIO structure will be studied in the near future.

\section{CONCLUSION}

Experimental results have shown that PS sourced beams have been successfully applied in the generation of millimetre and terahertz radiation. The output power of the devices has been estimated in the range of a few watts to a few tens of watts, which agrees well with simulations. This enables the PSsourced electron beam to be a very promising beam source for compact and cost-effective terahertz sources as well as not requiring the application of an external guide magnetic field to form and propagate the beam.

Various beam-wave interaction circuits have been studied covering a wide range of frequencies in W-band and G-band to investigate the potential application of PS generated electron beams. Several successful experiments have demonstrated the PS-sourced electron beam to be a very promising beam source in different beam wave interactions regions designed for the generation of terahertz radiation. Considering the merits of EIO, further studies of a planar EIO with a PS sheet electron beam will be conducted using this 
improved post-accelerated PS beam configuration and a higher frequency $200 \mathrm{GHz}$ EIO structure will be studied in the near future.

\section{ACKNOWLEDGEMENT}

This research was supported by the Engineering and Physical Sciences Research Council (EPSRC), UK.

\section{REFERENCES}

[1] H. Yin, G. R. M. Robb, W. He, A. D. R. Phelps, A. W. Cross and K. Ronald, "Pseudospark-based electron beam and Cherenkov maser experiments", Phys. Plasmas, vol. 7, no. 12, pp. 5195-5205, Dec. 2000.

[2] H. Yin, W. He, A. W. Cross, A. D. R. Phelps, and K. Ronald, "Singlegap pseudospark discharge experiments" J. Appl. Phys., vol 90, pp. 3212-3218, Oct. 2001.

[3] H. Yin, A. W. Cross, A. D. R. Phelps, D. Zhu, W. He, and K. Ronald, "Propagation and post-acceleration of a pseudospark-sourced electron beam”, J. Appl. Phys., vol.91, no. 8, pp. 5419-5422, Apr. 2002.

[4] H. Yin, A. W. Cross, W. He, A. D. R. Phelps, and K. Ronald, "Pseudospark experiments: Cherenkov interaction and electron beam post-acceleration", IEEE Trans. Plasma Sci., vol. 32, no. 1, pp. 233239, 2004.

[5] H. Yin, A. W. Cross, W. He, A. D. R. Phelps, K. Ronald, D. Bowes, and C. W. Robertson, "Millimeter wave generation from a pseudospark-sourced electron beam, Phys. Plasmas, vol 16, no. 6, 063105, June 2009.

[6] J. Zhao, H. Yin, L. Zhang, G. Shu, W. He, J. Zhang, Q. Zhang, A. D. R. Phelps, and A. W. Cross, "Influence of the electrode gap separation on the pseudospark-sourced electron beam generation," Phys. Plasmas, vol. 23, 073116, July 2016.

[7] W. He, L. Zhang, D. Bowes, H. Yin, K. Ronald, A. D. R. Phelps, and A. W. Cross, "Generation of broadband terahertz radiation using a backward wave oscillator and pseudospark-sourced electron beam," Appl. Phys. Lett., vol. 107, no. 13, 133501, Sept. 2015.

[8] D. Bowes, H. Yin, W. He, L. Zhang, A. W. Cross, K. Ronald, A. D. R. Phelps, D. Chen, P. Zhang, X. Chen and D. Li, "X-ray Emission as a Diagnostic from Pseudospark-Sourced Electron Beams", Nuclear Inst. and Methods in Physics Research B, vol. 235, pp. 74-77, Sept. 2014.

[9] D. Bowes, H. Yin, W. He, A. W. Cross, K. Ronald, A. D. R. Phelps, D. Chen, P. Zhang, X. Chen and D. Li, "Visualization of a PseudosparkSourced Electron Beam", IEEE Transaction on Plasma Science, vol. 42, no. 10, pp. 2826-2827, Oct. 2014.

[10] Y. Yin, W. He, L. Zhang, et al., "Preliminary design and optimization of a G-band extended interaction oscillator based on a pseudosparksourced electron beam," Phys. Plasmas, vol. 22, no. 7, pp. 073102-1073102-6, Jul. 2015.

[11] Y. Yin, W. He, L. Zhang, H. Yin, C. W. Robertson and A. W. Cross, "Simulation and Experiments of a W-band Extended Interaction Oscillator based on a pseudospark-sourced electron beam," IEEE Trans. Electron Devices, vol. 63, no. 1, pp. 512 - 516, Jan. 2016.

[12] J. Zhao, H. Yin, L. Zhang, W. He, Q. Zhang, A. D. R. Phelps, and A. W. Cross, "Experiments on W-band extended interaction oscillator with pseudospark sourced post-accelerated electron beam”, Phys. Plasmas, vol. 24, 060703, 2017.

[13] J. Zhao, H. Yin, L. Zhang, G. Shu, W. He, Q. Zhang, A. D. R. Phelps, and A. W. Cross, "Advanced post-acceleration methodology for pseudospark-sourced electron beam", Phys. Plasmas. vol. 24, no. 2, 023105, Feb. 2017

[14] J. Zhao, H. Yin, L. Zhang, G. Shu, W. He, A. D. R. Phelps, A. W. Cross, L. Pang, and Q. Zhang, "Study of the beam profile and position instability of a post-accelerated pseudospark-sourced electron beam", Phys. Plasmas ,vol. 24, 033118, 2017.

[15] G. Shu, W. He, L. Zhang, Y. Yin, J. P. Zhao, A. W. Cross, A. D. R. Phelps, "Study of a $0.2 \mathrm{THz}$ extended interaction oscillator driven by a pseudospark-sourced sheet electron beam," IEEE Trans. Electron Devices, vol. 63, no. 12, pp.4955-4960, Dec. 2016. 